\title{
Simulation of Leaf Water Status
}

\author{
Tian-tai GUO, Chong-chong GUO, Dong-sheng LI, Ai-jun CHEN, Ming-ming ZHAO
}

College of Metrological Technology and Engineering China Jiliang University Hangzhou, P.R China

\begin{abstract}
Water state of plants has an important effect on their growth. In this paper, water state of plant leaves were simulated using Fluent, a CFD simulation software, and principles of physiological structure of plant leaf and the water transportation were analyzed through combination of plant physiology and fluid dynamics. Firstly, a simulation model of plant leaves was established in Gambit, a preprocessor software for Fluent; after that, the model was simulated in Fluent; then the results were exported to Tecplot, a post-processing software; finally, three-dimensional data graphics of the distribution of velocity, temperature and pressure were obtained, and analysis on the simulation results were made. The result of this paper is helpful for the further study of plant leaf physiology and future water-saving irrigation.
\end{abstract}

Index Terms: Computational fluid dynamics; Simulation; Plant leaves; Water status

(C) 2012 Published by MECS Publisher. Selection and/or peer review under responsibility of the International Conference on E-Business System and Education Technology

\section{Introduction}

Water takes a lager portion in growing plants, and the water content of Protoplast accounts for about $80 \%$ to $90 \%$. Therefore, the plant water status has a significant influence on the growth of plants. China is faced with a very severe shortage of clean water, and high-efficiency water-saving irrigation will be the only practical choice for the sustainable development of her agriculture. However, to perform efficient irrigation, the knowledge of water status of the plants is essential. Efforts have been made to measure the water content and monitor the water circulation in plants, and there are several methods of plant water measurement, such as aircoupled ultrasounds[1], spectral reflectance of leaves[2][3], terahertz radiation[4], etc. But at present the research in this respect is rather insufficient due to the immense diversity of plants and the complexity of plant structures.

In recent years computer simulation has been given more and more attention for their powerful calculation capabilities, and it's especially convenient for applications where mathematical models are hard to obtain. The application of computer simulation in plant physiology is increasing, e.g. D. Luquet simulated the leaves temperature of cotton plants[5], T. Czaszejko perfomed Monte Carlo simulation for the determination of statistical distribution of water tree lengths[6], etc. Current research on water status of plants mainly focuses on photosynthesis and the mechanism of water movement, and it is in a relatively immature period. This paper studies the water flow in plants from the perspectives of fluid dynamics and computer simulation, and obtains 
some qualitative results to demonstrate the distribution of temperature, pressure and velocity in different parts of the plants, thus providing useful information on water status of plants.

\section{Mathematical model of water flow in plants}

Water flow in plants can be studies using the principles of fluid dynamics. Reynolds number is an important parameter characterizing fluid flow. Liquid flow pattern of the Reynolds number is calculated as:

$R_{e}=\frac{\rho u d}{\eta}=\frac{u d}{v}$

where $R e$ is Reynolds number, $u$ is the average velocity of the fluid, $\rho$ is the density of the liquid, $\eta$ is dynamic viscosity, $d$ is the diameter, $v$ is the kinematic viscosity, and $v=\rho / \eta$, the movement of water viscosity is $v=0.0131 \mathrm{~cm}^{2} / \mathrm{s}$.

According to fluid dynamics, the energy of viscous fluid can be expressed in the form of differential equations:

$d\left(\pi+\frac{p}{\rho}+\frac{V^{2}}{2}\right)-(v \Delta u d x+v \Delta v d y+v \Delta w d z)=0$

where $u, v$ and $w$ are the velocities in the directions of $x, y$ and $z$ of flow line respectively, $\rho$ is pressure, $V$ is the flow velocity in a micro segment of flow line.

For the case where only gravity exists as mass force, the form of (2) becomes

$d\left(z+\frac{p}{\gamma}+\frac{V^{2}}{2 g}\right)-\frac{v}{g}(\Delta u d x+\Delta v d y+\Delta w d z)=0$

Equation (3) represents the change of the total mechanical energy of the fluid along the flow lines for unit weight.

The mechanical energy of fluid in unit time per unit weight is $z+\frac{p}{\gamma}+\frac{V^{2}}{2 g}$, and the work which viscous force has done in unit time per unit weight is $d h w^{\prime}$. By performing integration along the same flow line, we obtain

$z_{1}+\frac{p_{1}}{\gamma}+\frac{V_{1}^{2}}{2 g}=z_{2}+\frac{p_{2}}{\gamma}+\frac{V_{2}^{2}}{2 g}+\int_{1}^{2} d h_{w}^{\prime}$

Equation (4) shows that the mechanical energy of the fluid along the same flow line in unit time per unit weight is always decreasing in a viscous fluid, and cannot maintain conservation, while for ideal fluid the total mechanical energy conservation is maintained, i.e. with no loss of mechanical energy. The loss represents the consumption of mechanical energy when viscous particles of fluid do force in overcoming viscous stress. When viscous fluid flows in steady lamina, the total mechanical energy is decreasing along the way regardless of how potential energy, kinetic energy and pressure energy transform, and is always flowing from the high mechanical energy area to low energy area.

\section{Numerical simulation of leaf water status}

Numerical simulation of leaf water status is performed using FULENT, a computational fluid dynamics software, and the steps taken are shown in Fig. 1

Sponsored by National Natural Science Fund project (No.60671052) 


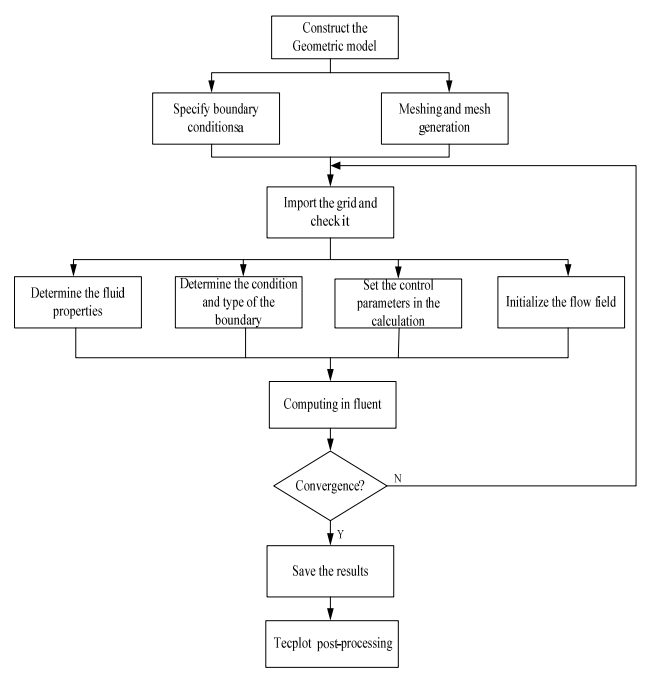

Figure 1. Figure1. Simulation process of leaf water status in FLUENT

To demonstrate the distribution of temperature, pressure and velocity in the plants, leaf blade with mesh structure is taken as an example here. Firstly, a simulation model of plant leaves was established in Gambit, a preprocessor software for Fluent, which is shown in Fig. 2. The main vein is modeled as a cylinder along the line $y=x$, with the length of $25 \mathrm{~mm}$. Offshoot 1 is parallel to the $y$-axis, starting from the point $(5 \mathrm{~mm}, 5 \mathrm{~mm})$ with the length of $10 \mathrm{~mm}$; Offshoot 2 is parallel to the $x$-axis, starting from the point $(10 \mathrm{~mm}, 105 \mathrm{~mm})$ with the length of $10 \mathrm{~mm}$. And the three columns were interconnected to form the whole body structure of a leaf. Fig. 2a shows the corresponding geometric model in Gambit. The inlet is at $\mathrm{x}=0 \mathrm{~mm}$, and three outlets are arranged, among which outlet 1 is at $y=15 \mathrm{~mm}$, outlet 2 is at the exit of the main vein, and outlet 3 is at $x=20 \mathrm{~mm}$. Fig. $2 \mathrm{~b}$ shows the designation of the inlet and outlets.

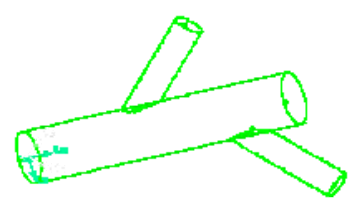

(a) Geometric model in Gambit

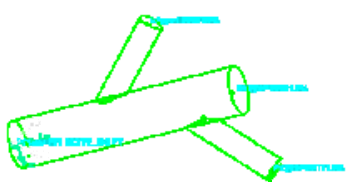

(b) Inlet and outlets

Figure 2. Figure2. Structure model of leaf blade in Gambit 
The wall mesh and exit planes are shown in Fig. 3, in which the meshes of plane $x=5 \mathrm{~mm}$, plane $y=15 \mathrm{~mm}$, plane $\mathrm{z}=0 \mathrm{~mm}$ and exit plane $1(\mathrm{y}=15 \mathrm{~mm})$ are shown.

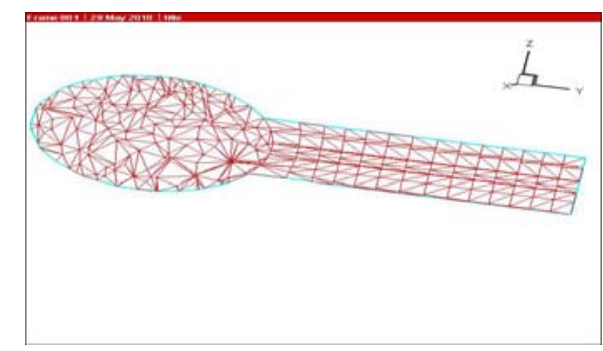

(a) Plane at $\mathrm{x}=5 \mathrm{~mm}$

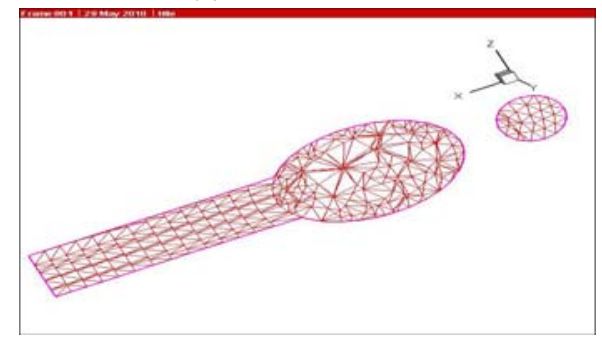

(b) Plane at $\mathrm{y}=10 \mathrm{~mm}$

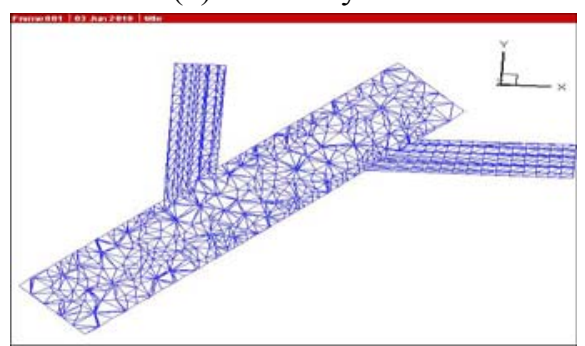

(c) Plane at $\mathrm{z}=0$ (top view)

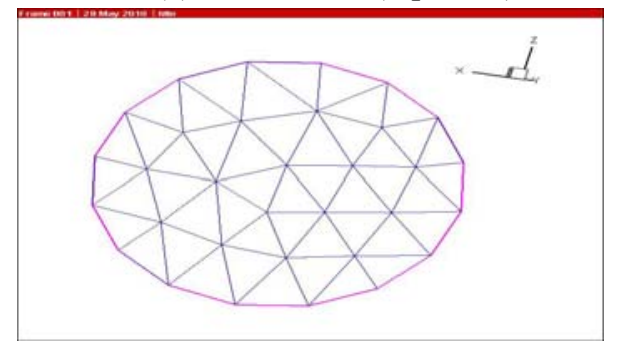

(d) Exit plane $1(\mathrm{y}=15 \mathrm{~mm})$

Figure 3. Wall mesh and exit planes of leaf blade

The results obtained from Fluent solver is shown in Figs. 4 and 5, among which Fig. 4 sets the four parameters of fluid flow in the Solution module of Fluent, and Fig. 5 shows the resulted residual plots. 


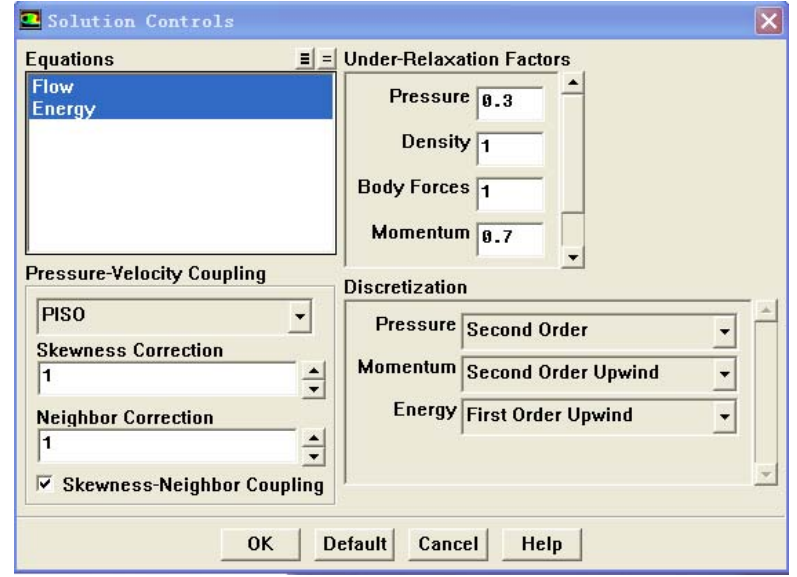

Figure 4. Settings of solving parameters

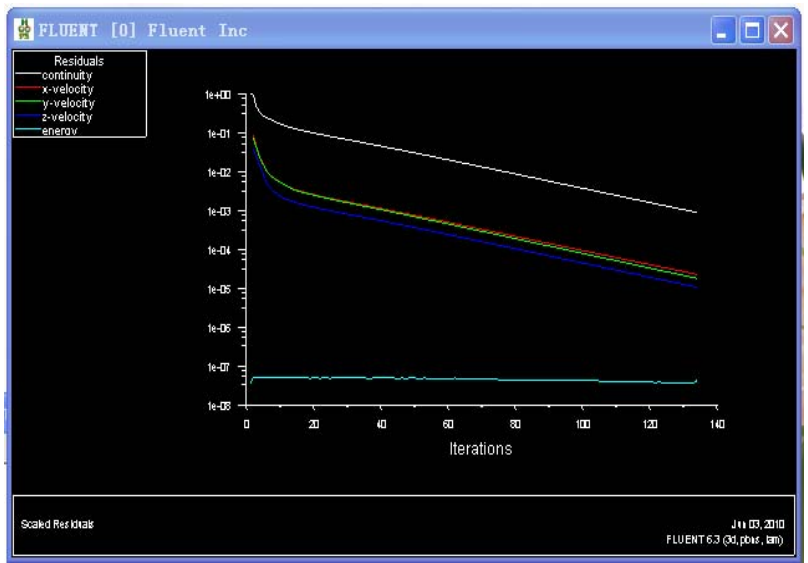

Figure 5. Residual plots

nsidering the fact that the physical structure of a plant leaf is rather complex, a relatively simple geometry model is established for a start. Then a structure grid of the corresponding model is created in the Gambit software after determining the type and properties of fluid. Finally, the appropriate simulation parameters and boundary conditions are set in Fluent, the simulation model is obtained. Simulaton is then performed in Fluent, and calculation results are obtained.

\section{4. ulation results and analysis}

The simulation results of Fluent is then exported to Tecplot, a post-processing software, and functions selected include velocity, pressure, temperature in the directions of $\mathrm{x}, \mathrm{y}, \mathrm{z}$. The display interface in Tecplot is selected, and the temperature, speed, pressure data graphics obtained are as shown in Figs. 6 to 8. 


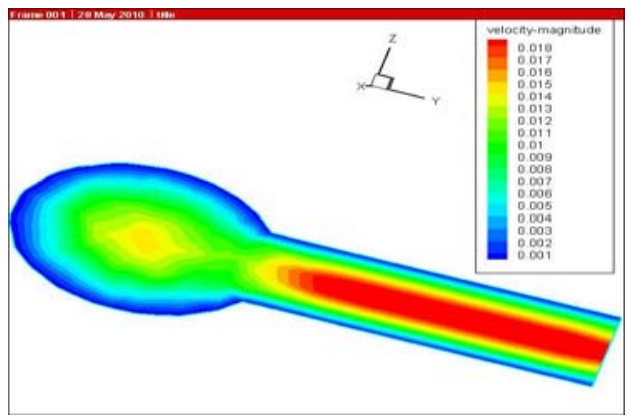

(a) Plane at $x=5 \mathrm{~mm}$

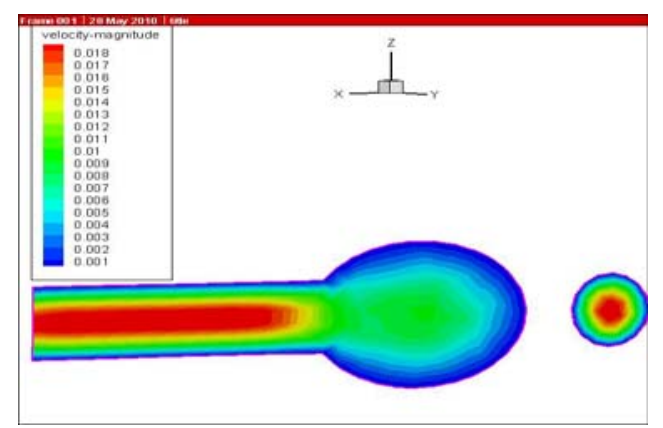

(b) Plane at $y=10 \mathrm{~mm}$

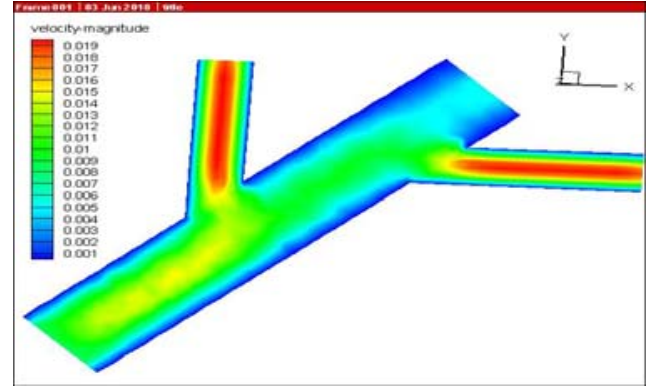

(c) Plane at $\mathrm{z}=0$ (top view)

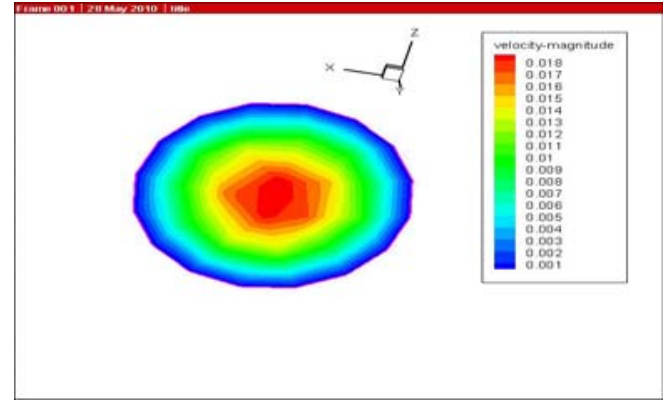

(d) Exit plane 1(y=15mm)

Figure 6. Velocity vectors 


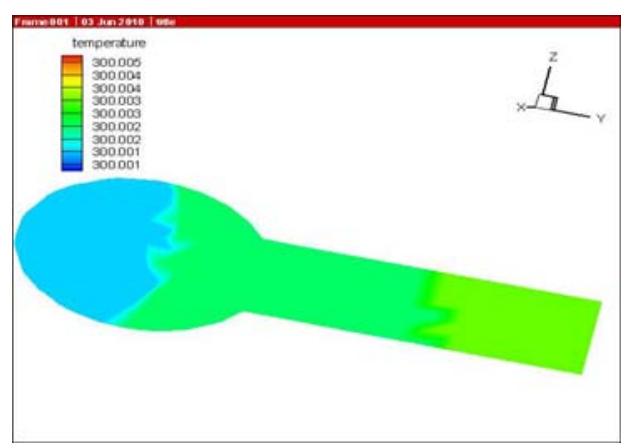

(a) Plane at $x=5 \mathrm{~mm}$

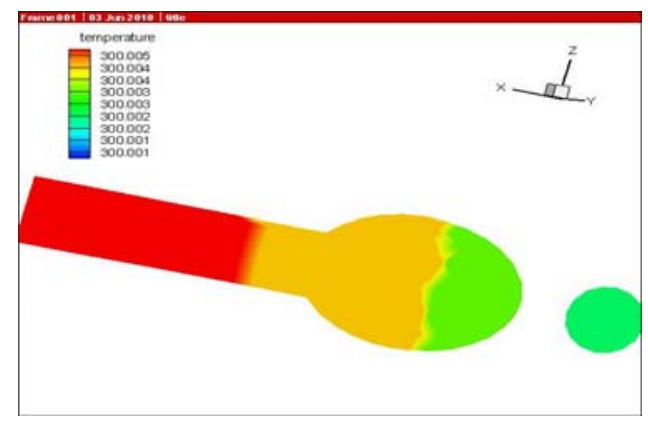

(b) Plane at $y=10 \mathrm{~mm}$

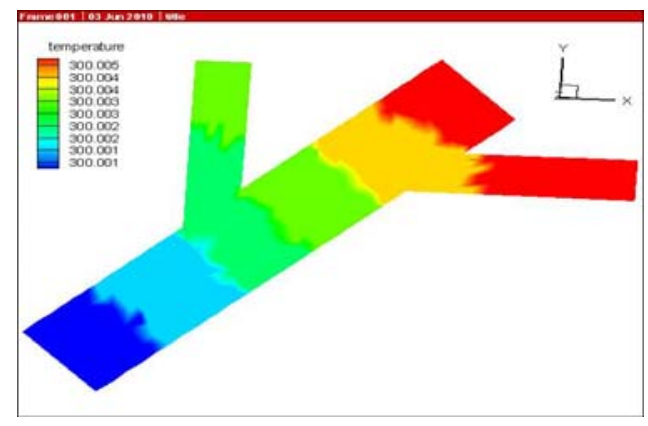

(c) Plane at $\mathrm{z}=0$ (top view)

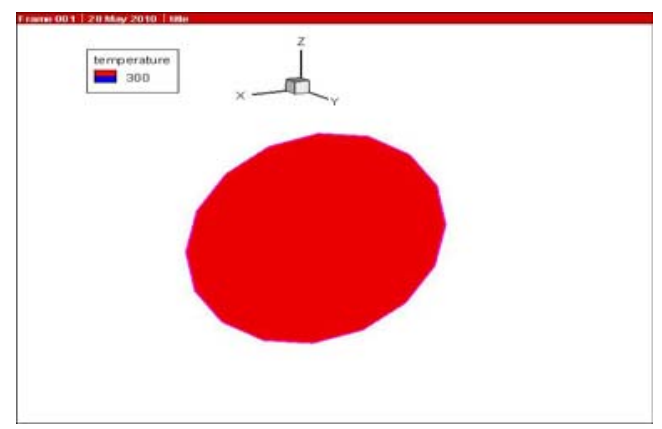

(d) Exit plane 1(y=15mm)

Figure 7. Temperature vectors 


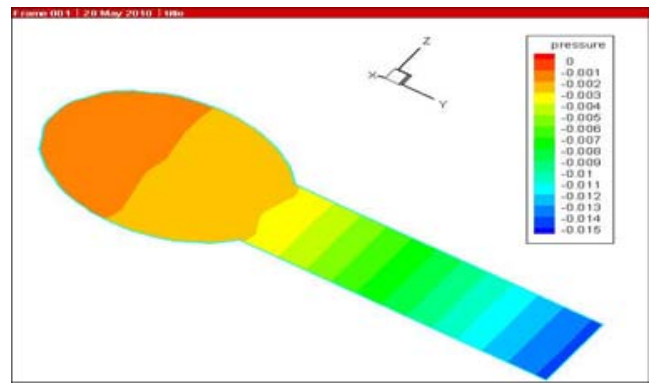

(a) Plane at $\mathrm{x}=5 \mathrm{~mm}$

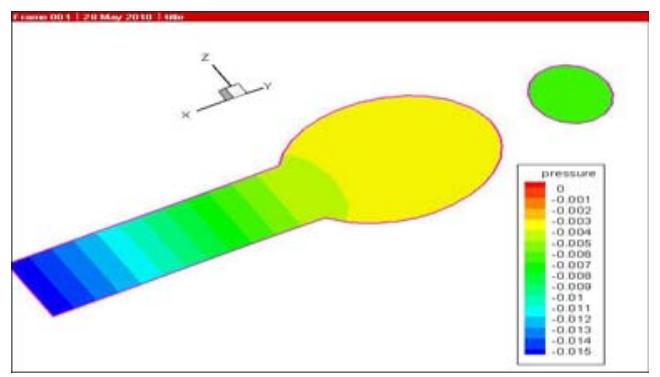

(b) Plane at $\mathrm{y}=10 \mathrm{~mm}$

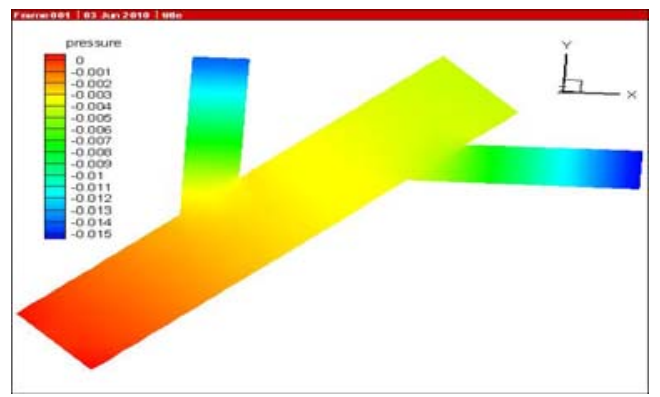

(c) Plane at $\mathrm{z}=0$ (top view)

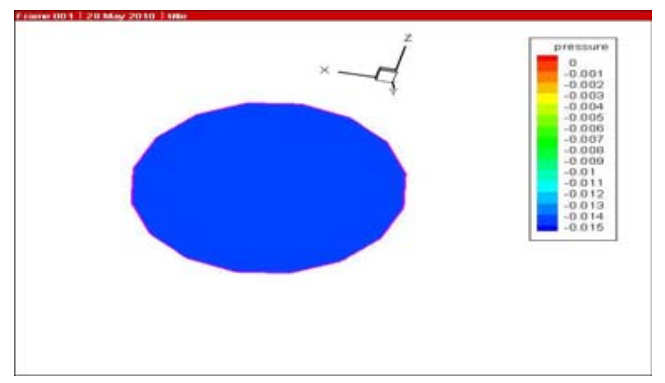

(d) Exit plane $1(\mathrm{y}=15 \mathrm{~mm})$

Figure 8. Pressure vectors

The flow of water inside the walls of plants is laminar flow, and the flow of water in the pipe along the direction parallel to the pipe axis is smooth linear motion. Fig. 6 shows that the fluid velocity is at the largest at the center of the pipe, at the smallest near the wall, and the velocity is decreasing along the direction of water 
flow. This is consistent with theory, because the laminar fluid's viscous force is much bigger than the inertial force, and a loss of mechanical energy is caused by viscosity. It can be seen from Fig. 7 that the water temperature in the leaf blade is getting higher along the direction of the water flow, which can be partly explained by the laminar flow of viscous water in the plant leaf. Fig. 8 shows that the pressure of plant leaf is at the maximum at the water entrance, and decreases along the direction of the water flow. It can also be seen from the simulation results that in the viscous fluid the mechanical energy of the fluid in unit time per unit weight decreases along a flow line, i.e. it's not conservative, and the energy loss represent the mechanical energy consumed by the work that the fluid particles do to overcome the viscous stress. Moreover, it can be seen that heat energy increases along the direction of the water flow for viscous fluid in steady laminar flow.

\section{Conclusions}

This paper studies the water status of plant leaves, and made analysis on the steady velocity field, temperature field and pressure field characteristics in a specific structure, and the results obtained are consistent with theoretical analysis. However, since the models adopted was a simplified one for the convenience of calculation, further study is needed to make it more applicable. For example, because of the permeability of leaf cells pipe, water pipe radius will change with the quality and speed of water flowing in it, and the geometry of dynamic mesh of a plant leaf should change with time, therefore a high-quality dynamic mesh model is needed, and the influence of such external factors as environment temperature, moisture, etc. should also be taken into consideration. The result of this paper is helpful for the further study of plant leaf physiology and future water-saving irrigation.

\section{References}

[1] D. Sancho-Knapik, J,J. Peguero-Pina and E,Gil-Pelegrín. Determination of Plant Leaves Water Status using Air-Coupled Ultrasounds [J]. IEEE International Ultrasonics Symposium Proceedings, september 2009, Vol 188, No. 2: 771

[2] Sophie Fabre, Audrey Lesaignoux. Influence of Water Content on Spectral Reflectance of Leaves in the 315- $\mu \mathrm{m}$ Domain[J]. International Conference on Conduction and Breakdown in Solid Dielectrics, June 2010, Vol 8, No. 1: 143

[3] Shudong Wang, Baozheng Chen. Research on Relation Between Water Evaporation and Spectral Curve of Leaves Based on Hyperspectral Reflectance[J]. International Conference on Conduction and Breakdown in Solid Dielectrics, July 2005,Vol 5:3125

[4] Sillas Hadjiloucas, Lucas S. Karatzas, John W. Bowen. Measurements of Leaf Water Content Using Terahertz Radiation[J]. IEEE Transactions On Microwave Theory And Techniques, February 1999,Vol 47, No. 2: 86-91M. Young, The Technical Writer's Handbook. Mill Valley, CA: University Science, 1989.

[5] D. Luquet, J. Dauzat. From the 3D modeling of cotton plants water balance to the simulation of leaves temperature[J]. International Conference on Conduction and Breakdown in Solid Dielectrics, july2000, Vol 1, No. 6: 366 [2]Sophie Fabre, Audrey Lesaignoux. Influence of Water Content on Spectral Reflectance of Leaves in the 3-15- $\mu \mathrm{m}$ Domain[J]. International Conference on Conduction and Breakdown in Solid Dielectrics, June 2010, Vol 8, No. 1: 143

[6] Tadeusz Czaszejko. Determination of Statistical Distribution of Water Tree Lengths: Monte Carlo Simulation[J]. International Conference on Conduction and Breakdown in Solid Dielectrics, 1998,Vol 3: 333 